cemoti $\begin{aligned} & \text { Cahiers d'études sur la Méditerranée } \\ & \text { orientale et le monde turco-iranien }\end{aligned}$

19 | 1995

Laïcité(s) en France et en Turquie

\title{
Laïcité, modernisme et islamisme en Turquie
}

Nilüfer GÖLE

\section{(2) OpenEdition}

\section{Journals}

Édition électronique

URL : http://journals.openedition.org/cemoti/1691

DOI : 10.4000/cemoti. 1691

ISSN : $1777-5396$

Éditeur

AFEMOTI

Édition imprimée

Date de publication : 1 janvier 1995

ISSN : 0764-9878

Référence électronique

Nilüfer GÖLE, "Laïcité, modernisme et islamisme en Turquie », Cahiers d'études sur la Méditerranée orientale et le monde turco-iranien [En ligne], 19 | 1995, mis en ligne le 14 mai 2006, consulté le 08 septembre 2020. URL : http://journals.openedition.org/cemoti/1691; DOI : https://doi.org/10.4000/ cemoti.1691

Ce document a été généré automatiquement le 8 septembre 2020.

Tous droits réservés 


\title{
Laïcité, modernisme et islamisme en Turquie
}

\author{
Nilüfer GÖLE
}

La laïcité sans la démocratie

1 Le renouveau des mouvements islamistes auquel on assiste dans la plupart des pays musulmans depuis la fin des années 70, renvoie à la question de savoir si l'islam est compatible avec la démocratie. Au fond, cette question revêt plusieurs dimensions. En premier lieu, l'islam en tant que religion est souvent considéré comme un obstacle au libéralisme politique. Or, on ne peut affirmer que la tradition judéo-chrétienne ait été pour ce dernier un obstacle moindre. Il faut distinguer analyse théologique et réalités politiques. En Occident, l'idéal démocratique s'est développé progressivement en engendrant une réforme de la religion. A la lueur des expériences des pays occidentaux, on doit ainsi reposer le problème de la relation entre l'islam et la démocratie en se demandant si le processus de modernisation et de démocratisation dans les pays musulmans n'entraîne pas fatalement une reformulation de l'islam ? En effet, d'une façon paradoxale, les mouvements islamistes contemporains ouvrent la voie à une nouvelle conception de l'islam, d'une part en critiquant ses interprétations traditionnelles, d'autre part en s'efforçant de développer une alternative à la société moderne. En second lieu, il faut également observer que la plupart des pays musulmans ont un pouvoir laïque mais vivent sous un régime autoritaire. Le problème de la compatibilité entre l'islam et la démocratie n'est donc pas indépendant des rapports de pouvoir qu'entretiennent dans ces pays élites occidentalisées et sociétés musulmanes. La nature autoritaire de la modernisation entamée dans beaucoup d'Etats musulmans depuis le XIXème siècle est sans doute à l'origine des déboires qu'a pu y connaître la démocratie le plus souvent.

2 L'existence d'un divorce politique et culturel entre une élite occidentalisée et un peuple resté très attaché à la religion a été la caractéristique la plus saillante de la vie politique des pays musulmans. De plus, la laïcité, condition sine qua non d'un pouvoir politique moderne, a accru cette fracture. Dans les pays musulmans qui ont connu une forte volonté politique de modernisation (Turquie, Iran...), on observe une 
contradiction intrinsèque entre laïcité et démocratie. Le principe de laïcité a primé rapidement sur le principe démocratique de souveraineté populaire et lorsque s'est produite une transition démocratique, elle a souvent été interrompue, car la participation des partis islamistes aux élections a été considérée par les élites, tant locales qu'occidentales, comme une stratégie déguisée de conquête du pouvoir à des fins théocratiques.

3 La modernisation turque et son histoire politique sont l'illustration même de cette contradiction. La Turquie qui s'est engagée très tôt, comme l'on sait, dans un processus radical de sécularisation, fut gouvernée par un parti unique de 1923 (fondation de l'Etat-Nation laïque et républicain) à 1946 (passage à un système pluraliste). Le principe de laïcité l'a emporté sur le pluralisme et la démocratie car les élites modernistes considéraient que la souveraineté du peuple risquait de provoquer le retour de l'Islam. Effectivement, avec le passage au pluralisme politique en 1946, l'organisation d'élections libres en 1950, les valeurs islamiques se sont plus facilement exprimées et ont été incarnées par le nouveau Parti démocrate parvenu au pouvoir. Mais, le coup d'Etat de 1960 a mis fin à ce premier processus de démocratisation et a renforcé l'alliance entre les élites "progressistes" et l'armée face au peuple musulman.

4 En d'autres termes, il y a une sorte de cercle vicieux dans le système politique des pays musulmans que l'on pourrait décrire de la façon suivante: d'abord, la laïcité est imposée par un régime autoritaire, ensuite, une transition démocratique favorise la liberté d'expression culturelle mais aussi le développement d'une action politique islamique, enfin, cette mutation est dénoncée par les élites occidentalisées. La boucle est bouclée et l'on assiste alors le plus souvent à une intervention militaire qui prétend restaurer l'ordre laïque. L'expérience algérienne est l'exemple le plus récent et le plus dramatique de cet enchaînement infernal : laïcité, démocratie, islam. Face au régime autoritaire du FLN, la montée du FIS a été portée au départ par les aspirations démocratiques de la société algérienne. Mais les élites laïques, inquiètes de la menace intégriste islamique ont préféré avoir recours à un coup d'Etat. Le processus de démocratisation a de fait été interrompu tandis que la naissance d'un espace politique véritable devenait impossible et que le pays sombrait dans l'illégalité et le terrorisme.

5 Autrement dit, la laïcité n'est pas un terme neutre, un principe exempt d'idéologie et insensible aux rapports de pouvoir. Elle contribue à définir et sous-tend en réalité le pouvoir politique et culturel des élites modernistes. Ainsi, la tension intrinsèque existant entre laïcité et démocratie relève des rapports de pouvoir entre les élites occidentalisées et la société musulmane. Parallèlement, l'affirmation de l'islam comme force politique s'opère par une réinterprétation et une exacerbation de l'identité et de la culture musulmanes. En ce sens, les mouvements islamistes fournissent l'un des leviers idéologiques de l'“empowerment" de la société.

La laïcité comme mode de vie

6 Pour mieux appréhender la signification sociologique des mouvements islamistes, il est important d'analyser le déroulement de la modernisation dans les pays musulmans. Cette dernière prend une forme très différente de celle qu'elle a pu avoir dans les pays occidentaux, dans la mesure où elle impose politiquement une occidentalisation des références culturelles, des modes de vie, des identités homme/femme. L'histoire turque de la modernisation est à cet égard exemplaire. C'est l'histoire d'une modernisation culturelle, voire d'une conversion à la "civilisation". En transformant un empire pluriethnique en Etat-Nation républicain et laïque, les réformes kémalistes se sont attachées 
aussi à promouvoir une conversion à la "civilisation" qui a pénétré les styles de vie, les comportements, l'éthique et l'esthétique, en bref "l'habitus", dirait Pierre Bourdieu1, de la femme et de l'homme musulmans. Même si le concept de "civilisation" recouvre un champ très large, allant des technologies aux habitudes de la table, il est surtout, comme l'a souligné Norbert Elias². Il exprime, face à des sociétés "non civilisées" tout ce qui fait la fierté de l'Occident: sa technologie, ses règles de savoir-vivre, sa connaissance scientifique, sa vision du monde et bien d'autres choses encore. En fait, le concept de "civilisation" incarne la supériorité du monde occidental et confère aux modèles culturels de ce dernier une dimension universelle.

7 Les efforts de modernisation dans un pays musulman deviennent une affaire de "civilisation" dans la mesure où les termes mêmes de cette modernisation sont définis par la pratique et la culture occidentales. Lorsqu'à la Renaissance, l'Europe et l'Occident s'imposent comme le lieu par excellence de l'innovation et de la modernité (un phénomène qui se poursuivra avec les Lumières, l'avènement de la société industrielle et qui se poursuit encore aujourd'hui), les expériences non-occidentales perdront de leur éclat historique et ne seront plus définies qu'en termes négatifs par des noms qui n'en sont pas ("non western", disent par exemple les Anglais). Comme l'écrit Daryush Shayegan ${ }^{3}$, ces sociétés sont abandonnées à la périphérie de la civilisation occidentale, exclues de l'histoire et de la connaissance car elles ne peuvent plus participer "au carnaval du changement" et cette exclusion dégénère en une "schizophrénie culturelle". Ce sont des sociétés à "faible historicité", c'est-à-dire des sociétés dont la capacité à produire la modernité en tant que développement endogène est faible ${ }^{4}$. Comme le dit H. Djait, leur histoire est celle de la permanence de leur tentative de modernisation, en fin de compte, un pâle reflet de l'histoire de l'Occident. La rencontre de l'Est et de l'Ouest conduit donc au déclin de l'identité islamique plutôt qu'à un échange réciproque entre deux cultures. En réalité, le concept de "civilisation" ici ne tient pas compte de la relativité historique des civilisations (civilisation asiatique, civilisation française, civilisation islamique...) mais il renvoie surtout à la supériorité de l'Occident. Les réformateurs turcs n'ont pas caché leurs intentions à cet égard. Leur objectif principal était "d'accéder à la civilisation contemporaine" (muasir medeniyet seviyesine erismek), c'est-à-dire dans leur esprit à la civilisation occidentale.

8 Le débat, qui apparaît déjà en Turquie à la fin du XIXème siècle à propos des limites et des modalités de l'occidentalisation, montre bien l'intérêt central de la question des rapports entre identité culturelle et modernisation. Pour les conservateurs de cette époque, les aspects matériels de la civilisation doivent être distingués de ses aspects spirituels, car les premiers consistent en une suite d'innovations technologiques, tandis que les seconds affectent l'identité religieuse et culturelle d'un pays. Dès lors, les réformes doivent se limiter pour eux au domaine de la science et de la technologie : de l'Ouest, pensaient-ils on peut emprunter l'aspect matériel, mais en prenant garde de préserver sa propre culture. Les occidentalistes, quant à eux, vont défendre l'idée selon laquelle la civilisation est un tout que l'on ne peut diviser et qu'en conséquence il faut adopter en bloc. Pour ces modernisateurs, elle n'est pas le résultat d'une culture et d'une religion spécifiques mais le produit universel de la science et de la rationalité, valable en tout lieu et en tout temps.

9 La modernisation turque découlera de la seconde conception de la civilisation. Le modernisme kémaliste de 1923 marque en effet définitivement la victoire du courant occidentaliste sur le courant conservateur. Cette définition universaliste de la 
modernisation se doublera d'une volonté politique d'effacer les traces de la culture religieuse. Pour les élites modernistes, le progrès social passe par le rejet des particularismes culturels locaux, il doit permettre de briser les chaînes de la culture islamique. L'Empire ottoman qui avait été l'étendard de l'islam en réussissant à réunir dans un même monde les centres musulmans les plus importants (Le Caire, Bagdad, Damas, La Mecque ou Médine) et en menaçant la chrétienté en son coeur pendant près de deux siècles, avait finalement assisté, impuissant, au basculement du centre de gravité du monde vers un Occident sûr de lui et dominateur. Il était devenu "l'homme malade de l'Europe" ${ }^{5}$ et son déclin avait entraîné son glissement d'une civilisation à une autre mais l'ironie de l'histoire allait être que le Turc qui avait symbolisé, aux yeux des Occidentaux, pendant des siècles, l'“autre", "le barbare" et pour tout dire le "musulman", voudra alors entrer dans le club des "civilisés" et que pour ce faire, il devra s'inventer des "barbares" et montrer du doigt les obstacles à la "civilisation" : hier les musulmans, aujourd'hui les Kurdes!

La Laïcité au féminin

10 C'est incontestablement pour le statut de la femme que le choix de la civilisation occidentale pose le plus de problèmes. Dans la société musulmane, la question de la femme ne se pose pas en termes de condition féminine, elle renvoie directement à des enjeux de société, à des choix touchant à la civilisation.

11 L'équation qui exprime le mieux l'histoire de la modernisation est celle qui rapproche "progrès du pays" et "émancipation des femmes". Dans ce registre, le kémalisme par son radicalisme est unique. C'est dans le domaine des relations homme/femme, et dans celui de la redéfinition des espaces privé et public que le choix de la civilisation occidentale est ressenti le plus fortement. Peut-être l'altérité la plus tenace entre le monde occidental et le monde musulman réside-t-elle dans la définition de la sphère du privé et de l'identité des femmes. Le privé, qui n'a pas de synonyme dans la culture musulmane, renvoie à la sphère close, à l'intimité mais est aussi un espace sexué, délimité par l'interdit des femmes, mieux exprimé par le mot "mahrem". Le voile islamique rappelle l'interdit du regard (de l'homme extérieur à la famille) sur la femme, mais il marque également la frontière entre dedans et dehors, entre l'intérieur et l'extérieur et symbolise la ségrégation des sexes. Or, le réformisme kémaliste, pour user ici d'une métaphore, cherchera justement à détruire ce mahrem et à projeter la femme dans la vie publique. La faculté de voir les femmes mettra alors en évidence un changement de civilisation car cette visibilité des femmes sera à la fois corporelle (abandon du voile), urbaine et publique (mixité) ou politique (droit de vote et éligibilité accordée aux citoyennes turques en 1934). Enfin, la suppression de la Sharia et l'adoption du code civil suisse en 1926 vont permettre de consacrer le principe de laïcité en assurant en fait la soumission des relations entre hommes et femmes à un système juridique moderne. Autrement dit, si l'impact le plus visible de la modernisation touche les structures étatiques et les institutions politiques, ses effets a priori les plus diffus mais en réalité les plus profonds concernent le niveau "civilisationnel", notamment le capital symbolique, la construction des identités, la définition de l'éthique et de l'esthétique. La distinction entre les manières "civilisées" et "non-civilisées" sera réglée dans les moindres détails par les modernistes. Le mode de vie "à la franca" sera loué tandis que le mode de vie "à la turca" sera délaissé. Porter une cravate et un chapeau, se raser les moustaches, se promener en couple, aller au théâtre ou au bal, écrire de gauche à droite, manger avec une fourchette, tels seront désormais les attributs de l'individu "civilisé" et "progressiste". Les valeurs esthétiques 
connaîtront également une mutation. Les canons de la beauté orientale : rondeurs, vêtements amples, cheveux longs, seront supplantés par l'image d'une femme occidentale mince, énergique, active, portant le plus souvent les cheveux courts. Ainsi le style de vie occidental va prendre le pas sur son homologue oriental.

12 Dans un tel contexte, la mode sera le cheval de Troie d'un mouvement exaltant une nouvelle vie, une nouvelle "civilisation". Chaises, gramophones, pantalons ou corsets, vont symboliser la vie "à la franca" et favoriser la formation de nouveaux comportements en influant sur les habitudes et les divertissements des Turcs. Si l'on suit Georges Simmel, lorsqu'il déclare que la manière de se vêtir agit directement sur les comportements et les gestes d'une personne, on peut penser alors que des habits semblables peuvent générer une conduite semblable ${ }^{6}$. Avec la mode, en fait, le style de vie occidental entrera dans l'environnement musulman en transformant des habitudes vestimentaires qui étaient liées avant tout aux traditions et reflétaient des différences ethniques et sociales. La mode, en donnant un sens positif au changement et à la nouveauté, va favoriser la prise en compte du moment présent et changer l'environnement musulman qui restait justement hors du temps et monotone.

13 Le changement introduit dans le goût ne sera pas non plus chose innocente. Il accentuera le glissement d'une civilisation à l'autre. En outre, le goût, facteur de distinction sociale, établira une hiérarchie sociale, une stratification sociale ${ }^{7}$. Par conséquent, il se créera implicitement, en l'occurrence, des rapports de pouvoir entre les élites occidentalistes et la société musulmane, rapports dont les enjeux seront définis en termes culturels, par la distinction sociale. L'islamisme radical contemporain révèle bien ce rapport de pouvoir dans sa forme exacerbée. La réapparition du voile islamique donne à voir le conflit social sous l'angle de la civilisation : c'est l'irruption des acteurs musulmans sur la scène historique avec leurs propres éthique et esthétique, le retour de ceux qui ont été tenus à l'écart du progrès et de la rationalité.

14 En fin de compte, le renouveau islamique représente un défi pour l'équation établie par les élites modernistes entre "civilisé" et "occidentalisés". Pour comprendre la signification de l'islamisme aujourd'hui, il faut reconsidérer les enjeux de civilisation que constituent des symboles corporels comme le voile pour les femmes et la barbe pour les hommes ou des interdits moraux comme la chasteté, l'homosexualité ou la consommation d'alcool. Le mode de vie, et plus particulièrement la réglementation sociale des rapports entre hommes et femmes, devient ainsi un enjeu central qui détermine les rapports de pouvoir entre les islamistes et les laïques.

Les élites islamistes et la laïcité interdite

15 Le renouveau islamiste qu'ont connu les pays musulmans dans les années soixante-dix et quatre-vingt a été le plus souvent considéré et expliqué comme un activisme politique visant à instaurer un ordre islamiste basé sur la loi religieuse (la Sharia). Certes, c'est la stratégie politique qui est la plus visible au sein des multiples tendances de l'islamisme contemporain, mais dans l'observation de ce phénomène, il ne faut pas omettre de relever l'impact social que peuvent avoir l'appropriation d'une référence islamiste et la participation de nouveaux groupes sociaux qu'elle génère. L'idée d'une révolution islamique conçue comme une révolte des masses est en effet tellement énorme que cette logique politique de révolution ne peut que cacher une logique qui est en réalité socio-culturelle.

16 Dès lors, il est difficile de voir dans le phénomène islamiste qu'une réaction pathologique à un ordre social en crise. En tant que mouvement social, ce phénomène 
participe en effet aux tentatives de résolution de cette crise $^{8}$. En conséquence, les acteurs islamistes ne peuvent être qualifiés à la fois de "masses populaires" et de "militants activistes" car les mouvements islamistes génèrent une élite socioprofessionnelle et culturelle (une contre-élite). Les militants des mouvements islamistes sont souvent recrutés au sein des universités, ce qui montre bien que ce n'est pas un mouvement issu d'une population traditionnelle mais plutôt un mouvement citadin investi par des groupes sociaux récemment urbanisés et scolarisés. Les acteurs de l'islamisme turc ont en fait profité des opportunités que leur ont offert le pluralisme politique et surtout le développement de l'économie de marché au cours de la décennie 1983-1993.

17 On peut avancer l'idée que les mouvements islamistes évolueront vers une logique de participation s'il y a une possibilité de renouvellement des élites par le système éducatif, par la mobilité sociale ascendante et par la participation démocratique. Le cas turc pourrait être l'illustration de cette hypothèse. En revanche, dans les pays où les voies de la mobilité sociale et de la participation politique sont bloquées, les mouvements islamistes risquent de s'orienter irrémédiablement vers une logique d'exclusion et de violence.

18 Dans la période postérieure à 1983, en Turquie, on a vu des ingénieurs islamistes accéder aux rouages du pouvoir politique ou des étudiantes musulmanes voilées apparaître sur les campus des universités dans les grandes villes. La floraison de publications, de revues, de journaux d'obédience islamiste a par ailleurs permis de constater que ce phénomène avait un impact indiscutable sur les intellectuels. Ingénieurs, femmes voilées, intellectuels, ces nouveaux acteurs de l'islam vont sortir ce dernier de la périphérie du système. Les mouvements islamistes contemporains sont désormais au coeur de la modernité. Cela est vrai d'un point de vue géographique car ils sont bien dans les centres urbains, mais cela est également vrai d'un point de vue politique et culturel dans la mesure où ils participent à la production des valeurs et à l'orientation du changement. Leur mode d'action ne s'inscrit pas dans une logique de rupture avec le système mais dans une logique de participation. L'élite islamique actuellement en voie de formation aspire au contrôle de la modernité plutôt qu'à son rejet.

19 Les trois figures-types que nous évoquions - ingénieurs, intellectuels, femmes reflètent aussi tout à fait les termes du débat sur la laïcité qui traverse l'islamisme. Les ingénieurs sont à la fois tentés par la rationalité et par la foi, les intellectuels sont écartelés entre l'esprit critique et la moralité islamique, les femmes sont attirées par des aspirations individualistes et par une logique communautaire. Dans la mesure où la rationalité, la pensée critique et l'individualisme vont constituer un pôle indépendant de référence pour ces trois catégories d'élites islamiques formées au demeurant par l'enseignement laïc, on peut considérer qu'un processus de sécularisation est en route. En effet, plus ces gens se forgent une identité professionnelle en tant qu'ingénieur ou qu'intellectuel, plus le domaine du profane sera chez eux séparé du domaine du sacré. Mais cette séparation des domaines, cette sécularisation, ne sont pas voulues. Elles créent des tensions et sont les germes des conflits à venir à l'intérieur du mouvement islamiste. Les aspirations individualistes des femmes, l'esprit critique des intellectuels, la rationalité pragmatique des ingénieurs défient en fait un projet islamiste basé sur le caractère sacré de la conviction et sur l'appartenance à la communauté des croyants. D'un côté, l'idéologie islamiste défend un mode de vie islamique inséparable de 
l'identité islamique contre les projets modernisateurs, de l'autre, la pratique sociale des élites islamistes se développe en référence aux problèmes de sécularisation et par là même questionne, met à l'épreuve, l'idéologie islamiste.

Islamisation de l'espace public ou sécularisation des pratiques?

20 L'islamisme contemporain se forge dans le conflit potentiel entre le néocommunautarisme autoritaire et la communauté imaginaire "identitaire". En effet, l'islamisme plus qu'une idéologie politique est une communauté imaginaire, c'est-àdire un lieu où l'on rêve de relations profondes et horizontales entre des personnes qui ne se connaissent pas ${ }^{9}$, un lieu relevant du domaine du sacré. Or, si l'individu s'affirme de par sa pratique même par la sécularisation, c'est-à-dire par la séparation des domaines du sacré et du profane, l'islamisme aspire à une communauté totale qui trouve sa raison d'être dans l'Etat islamique.

21 Les analyses de l'islamisme ont mis effectivement l'accent sur ce dernier aspect, sur l'unité de la religion et de l'Etat dans l'islam et elles en concluent le plus souvent que la Sharia est la source d'autoritarisme. Cette vision en elle-même est très "politiste". Elle met en lumière l'islam politique et définit la Sharia comme le contraire de la laïcité entendue comme principe de doctrine politique. Pour notre part, nous avons essayé de montrer comment la laïcité en pays musulman prenait le plus souvent une tout autre forme en définissant une nouvelle hiérarchie sociale, une nouvelle organisation sociale, et de nouveaux rapports entre les sexes. Nous avons aussi essayé de situer à la fois la laïcité modernisatrice et l'islamisme politique par rapport à la société et aux conduites sociales. On comprend pourquoi la question de la femme, pierre angulaire de l'organisation sociale ${ }^{10}$, n'est pas dans notre analyse une question secondaire ne relevant que de la condition féminine, mais une question de civilisation. Quant à la sexualité, aux rapports entre hommes et femmes et aux moeurs en général, ce ne sont pas non plus des épiphénomènes de l'islamisme. On ne peut comprendre les tensions existant entre la laïcité et l'islamisme si l'on refuse de voir qu'il y a là des enjeux qui touchent à la définition même des modes de vie.

22 Ainsi, il faut dépasser les frontières de l'analyse politique pour s'intéresser à l'individu, à la délimitation des espaces privé et public, aux questions d'éthique et d'esthétique. Le contrôle de l'espace public devient alors un des enjeux centraux de la démocratie et du pluralisme politique. En effet, la politique islamiste reconquiert des individus qui étaient restés jusqu'alors en marge de la vie politique. Cette repolitisation engendre aujourd'hui des débats qui sont caractéristiques, sur la sexualité (affaire du foulard, mixité dans les autobus, sport des filles à l'école), sur l'art (statue d'Ankara considérée par le maire comme obscène) ${ }^{11}$, sur les antennes paraboliques (intrusion de la culture occidentale dans les foyers), sur l'alcool (faut-il interdire la consommation d'alcool sur les terrasses des cafés à Beyoglu) ${ }^{12}$, sur le Nouvel an (y a-t-il là une pratique chrétienne ou pas?) ou sur le patrimoine historico-culturel turc (les murailles byzantines d'Istanbul ou les pyramides d'Egypte en font-elles partie ?). Tous ces exemples posent en fait le problème de la frontière entre le privé et le public, entre la religion et la politique, entre le passé et le présent, entre l'identité et la communauté. Plus que l'islam d'Etat c'est ici l'islamisation de l'espace public qui est en jeu.

23 Le contrôle de l'espace public, les droits de la majorité, le protectionnisme culturel sont les principaux axes politiques d'un néo-communautarisme autoritaire islamiste. En réalité, le débat qui s'organise autour de ces différentes questions permet de 
distinguer d'un côté des politiques libérales d'ouverture, et de l'autre des politiques autoritaires de fermeture.

Pour les islamistes toute la question est de savoir si ces trois piliers de l'islam que sont la foi, le mode de vie, et l'Etat vont parvenir à s'unir ou au contraire demeurer autonomes. Peut-on espérer voir se développer un communautarisme identitaire acceptant le pluralisme politique et éloignant le spectre d'un communautarisme autoritaire qui serait assuré par un Etat islamique? L'islamisme et le libéralisme politique peuvent-ils cohabiter? De nouvelles approches de la philosophie politique concernant le libéralisme et la définition de la laïcité, comme celles de Charles Taylor, s'efforcent d'ouvrir un espace pour les communautés et le multi-culturalisme. Elles peuvent nous fournir de nouvelles pistes et un nouveau langage pour une approche pertinente de l'islamisme ${ }^{13}$. En fin de compte, l'avenir de la démocratie dépendra beaucoup des capacités intellectuelles et politiques à lier identité collective et identité individuelle, communautarisme identitaire et libéralisme politique, et à assurer l'autonomie de l'espace public vis-à-vis du pouvoir étatique, que ce dernier soit laïque ou islamique.

\section{NOTES}

1. Pierre Bourdieu, La distinction (critique sociale du jugement), Paris, Ed. de Minuit, 1979.

2. Norbert Elias, La civilisation des moeurs, Paris, Calmann Lévy, 1973.

3. Daryush Shayegan, Le regard mutilé, Paris, Albin Michel, 1989.

4. Nous utilisons le concept de faible historicité afin de conceptualiser la problématique du développement au niveau des rapports sociaux, de l'action sociale, donc au niveau sociologique plutôt qu'économique.

5. John K. Esposito, The Islamic Threat, Myth or Reality, New York, Oxford Un. Press, 1992, p. 173.

6. Georg Simmel, Philosophie de la modernité, Paris, Ed. Payot, 1989.

7. Pierre Bourdieu, op. cit.

8. Alain Touraine, La voix et le regard, Paris, Ed. du Seuil, 1989.

9. Benedict Anderson, Imagined Communities (Reflections on the Origin and Spread of Nationalism), Thetford, Thetford Press, 1983.

10. Nilüfer Göle, Musulmanes et modernes, voile et civilisation en Turquie, Paris, Ed. de la Découverte, 1993.

11. Cf. dans cette livraison (entretien de Rusen Çakir avec Jean Marcou).

12. Ibid.

13. Charles Taylor, "The Politics of Recognition", Multiculturalism, ed. by Amy Gutman, Princeton, Princeton Un. Press, 1994. Egalement cf. "Communitarianism and Liberalism", Critical Review, vol. 8, n², Spring 1994. 